\title{
Pulsed radiation from neutron star winds
}

\author{
J. G. Kirk ${ }^{1}$, O. Skjæraasen ${ }^{1}$, and Y. A. Gallant ${ }^{2}$ \\ 1 Max-Planck-Institut für Kernphysik, Postfach 1039 80, 69029 Heidelberg, Germany \\ 2 Service d'Astrophysique, CEA-Saclay, 91191 Gif-sur-Yvette, France
}

\author{
Received 22 February 2002 / Accepted 17 April 2002
}

\begin{abstract}
The radiation of a pulsar wind is computed assuming that at roughly 10 to 100 light cylinder radii from the star, magnetic energy is dissipated into particle energy. The synchrotron emission of heated particles appears periodic, with, in general, both a pulse and an interpulse. The predicted spacing agrees well with the Crab and Vela pulse profiles. Using parameters appropriate for the Crab pulsar (magnetisation parameter at the light cylinder $\sigma_{\mathrm{L}}=6 \times 10^{4}$, Lorentz factor $\Gamma=250$ ) agreement is found with the observed total pulsed luminosity. This suggests that the high-energy pulses from young pulsars originate not in the corotating magnetosphere within the light cylinder (as in all other models) but from the radially directed wind well outside it.
\end{abstract}

Key words. pulsars: general - pulsars: Crab - MHD - radiation mechanisms: non-thermal

\section{Introduction}

Recently, the non-axisymmetric "striped pulsar wind" investigated by Coroniti (1990) and Michel (1994) has been reexamined (Kirk \& Lyubarsky 2001). A striped wind is a structure similar to a Parker spiral, produced by the outward radial advection of magnetic field lines with foot points anchored on the surface of the rotating neutron star. If the field lines originate from the poles of an obliquely rotating dipole, there exists a region around the equatorial plane where the polarity of the field at a fixed radius reverses at the rotation period, producing stripes of alternating magnetic field direction. Magnetic energy is dissipated into particle energy if reconnection occurs at the stripe boundaries. Coroniti (1990) proposed that this kind of dissipation proceeds at a rate sufficient to maintain the thickness of the current sheet approximately equal to the gyro-radius of the heated particles. Michel (1994) used essentially the same criterion, phrased in terms of the velocity of the current carriers. These early papers concluded that observations of the Crab Nebula, which indicate conversion of Poynting flux to particle-borne energy flux within $10^{9} r_{\mathrm{L}}$, (where $r_{\mathrm{L}}$ is the radius of the light cylinder) could be explained by this mechanism. However, Lyubarsky \& Kirk (2001) point out that reconnection is accompanied by acceleration of the wind. Both processes occur on the same timescale in the Coroniti/Michel approach, which is also the timescale on which the plasma expands. As a result, time dilation in the accelerated flow significantly reduces the dissipation rate. In the case of the Crab, conversion of Poynting flux to particle-borne energy

Send offprint requests to: J. G. Kirk, e-mail: john.kirk@mpi-hd.mpg.de flux is not achieved until $10^{12} r_{\mathrm{L}}$ - too slow to account for the observations (the $\sigma$-problem; see Melatos 1998).

However, our present understanding of the physics of the dissipation process does not exclude the possibility that it proceeds much more rapidly than suggested by Coroniti and Michel. Recent investigations of reconnection in a relativistic pair plasma indicate that the timescale is related to the crossing time of a fast magnetosonic wave (Zenitani \& Hoshino 2001). In the case of a transsonic wind, the process could then be completed within a few pulsar periods, giving the plasma insufficient time to accelerate to high Lorentz factor. If rapid reconnection in fact occurs, it will be accompanied by radiation losses (Melatos 1998) that may give rise to an observable signature. In this letter we adopt this hypothesis. We assume that dissipation is triggered at a surface $r=r_{0}$ well outside the light cylinder in the supersonic MHD wind of a pulsar and takes place rapidly. Using two simple models of the electron distribution we show (i) that for the winds of young pulsars (e.g., the Crab) the predicted emission is pulsed, with, in general, a pulse and interpulse that are not symmetrically spaced in phase and (ii) that the predicted synchrotron luminosity in the pulses agrees to order of magnitude with that observed in the high energy emission of the Crab pulsar.

Our calculations suggest that the high energy pulses emitted by young pulsars originate as synchrotron emission in the pulsar wind, far outside the light cylinder. This is in marked contrast with conventional models of high-energy pulse production at an inner or outer gap (for a review see Harding 2001) and also with magnetospheric synchrotron based models (Machabeli et al. 2000; Crusius-Wätzel et al. 2001). The current sheet just beyond 


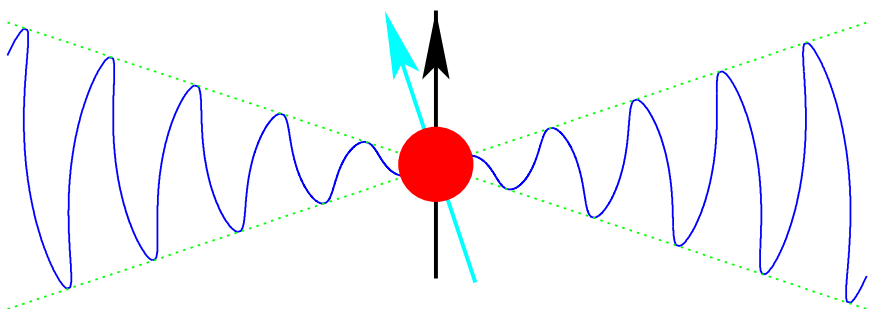

Fig. 1. A meridional section of the current sheet of an oblique rotator with a radial wind. The thin lines limiting the extent of the sheet in latitude depict the plane of the magnetic equator when the magnetic axis lies in the plane of the section.

the region of closed field lines in the magnetosphere has been proposed as the source of high energy radiation from the Crab pulsar (Lyubarsky 1996). But, in common with the others, this model relies for pulse production on the corotation with the star of a beam of radiation emitted from within the light cylinder.

\section{Geometry of the emission region}

Any radial, relativistic flow containing a periodic modulation of the emissivity is likely to appear pulsed to an observer because of two effects. Firstly, the strong Doppler boosting of approaching parts of the flow means that only a small cone of the flow is visible, propagating within an angle of roughly $1 / \Gamma$ with respect to the line of sight, where $\Gamma=\left(1-\beta^{2}\right)^{-1 / 2}$ is the Lorentz factor and $c \beta$ the radial speed. The spread in arrival times at the observer of photons emitted at the same radius but from different parts within this cone is roughly $r /\left(c \Gamma^{2}\right)$. Secondly, if emission is restricted to a range $\Delta r$ in radius, the fact that the photons move only slightly faster than the flow when seen in the laboratory frame means that the spread in arrival times is roughly $\Delta r /\left(c \Gamma^{2}\right)$. Thus, for a wind modulated at the period $P=2 \pi r_{\mathrm{L}} / c$ of a rotating star, the approximate condition for the observation of pulsed emission is

$\Delta r, r \lesssim \Gamma^{2} r_{\mathrm{L}}$

If this is fulfilled, the observed pulse shapes appear similar to the pattern of modulation of the flow. In particular, for a flow containing the corrugated spiral current sheet of an oblique rotator (Fig. 1), there should in general be two components (pulse and interpulse) which are symmetrically placed if the pulsar is viewed from the equatorial plane, but are asymmetrical when viewed from higher latitudes. Denoting by $\alpha$ the angle between the rotation axis and the magnetic axis, and by $\zeta$ the angle between the rotation axis and the viewing direction, these pulses appear only for $\left|90^{\circ}-\alpha\right|<\zeta<180^{\circ}-\left|90^{\circ}-\alpha\right|$.

To improve on the estimate (1) and calculate the expected pulse shapes we adopt the following model of the volume emissivity $\epsilon$ of the wind:

(i) The emission region is located on the current sheet in the supersonic MHD wind of a rotating neutron star with an oblique, split-monopole magnetic field. (ii) The dependence on frequency $\nu$ of the volume emissivity is a power law: $\epsilon \propto \nu^{-a}$.

(iii) The emission switches on abruptly as plasma in the sheet crosses the surface $r=r_{0}$. Thereafter the volume emissivity decays as a power law in radius: $\epsilon \propto r^{-2-q}$.

Outside the light cylinder, assumption (i) should be a good approximation, independent of the actual configuration of the magnetic field close to the pulsar surface. The assumption that emission occurs only at the exact position of the current sheet is an idealisation - in reality, the entire flow may be modulated, making the observed pulse more complex. In this case, our computations provide the Green's function with which the pattern is to be folded to find the observed pulse shape. If the wind carries enough particles to ensure MHD behaviour at least initially (for an alternative approach see Michel \& Li 1999), a simple solution is available in the ultra-relativistic limit (Bogovalov 1999). In it, the velocity is radial and constant and the magnetic field is almost toroidal. The thin current sheet in such a wind is described by the equation

$$
\begin{aligned}
r & =r_{\mathrm{s}}(\theta, \phi, t) \\
& =\beta r_{\mathrm{L}}\left[ \pm \arccos (-\cot \alpha \cot \theta)+c t / r_{\mathrm{L}}-\phi+2 n \pi\right]
\end{aligned}
$$

(Kirk \& Lyubarsky 2001), where $(r, \theta, \phi)$ are polar coordinates, $t$ is the observer-frame time and $n$ is an integer.

Assumption (ii) ensures the pulse shape is independent of frequency. In reality, the electron distribution may exhibit breaks and cut-offs, which translates into different pulse shapes at different frequencies. Our assumption, however, ensures that all parts of the flow can contribute to the emission at a given frequency, making it harder to obtain sharply defined pulses.

The dependence of the observed flux $F$ on $t, \alpha$ and the direction of observation $\hat{\boldsymbol{n}}$ is:

$$
\begin{aligned}
F \propto & \int_{-\infty}^{+\infty} \mathrm{d} t^{\prime} \int_{r_{0}}^{\infty} \mathrm{d} r \int_{90^{\circ}-\alpha}^{90^{\circ}+\alpha} \mathrm{d} \theta \int_{0}^{2 \pi} \mathrm{d} \phi \\
& r^{-q} D^{2+a} \delta\left[r-r_{\mathrm{s}}\left(\theta, \phi, t^{\prime}\right)\right] \delta\left(t^{\prime}-t+\hat{\boldsymbol{n}} \cdot \boldsymbol{r} / c\right)
\end{aligned}
$$

where $D=1 /(1-\beta \hat{\boldsymbol{r}} \cdot \hat{\boldsymbol{n}})$ is the conventionally defined Doppler factor, apart from a (constant) factor of $\Gamma$. Choosing $a=0$ and $q=3$, which is appropriate for the synchrotron radiation of a fixed number of particles that undergo predominantly adiabatic losses in the expanding plasma, we present sample pulses for various Lorentz factors in Fig. 2. For the higher Lorentz factors this figure also displays the effect of a faster fall-off of the emissivity towards higher radii $(q=6)$, mimicking the effect of an accelerating wind. The asymmetry in time of the pulses is produced by our assumption of a sudden switching on of the emission and is reduced by a more rapid switching off. The parameters of this figure have been chosen to correspond to the Crab pulsar. The radio emission of this object is complex. The shape of the high energy pulses is repeated in the radio, suggesting a common emission site, which in our model would be the wind. But there are 

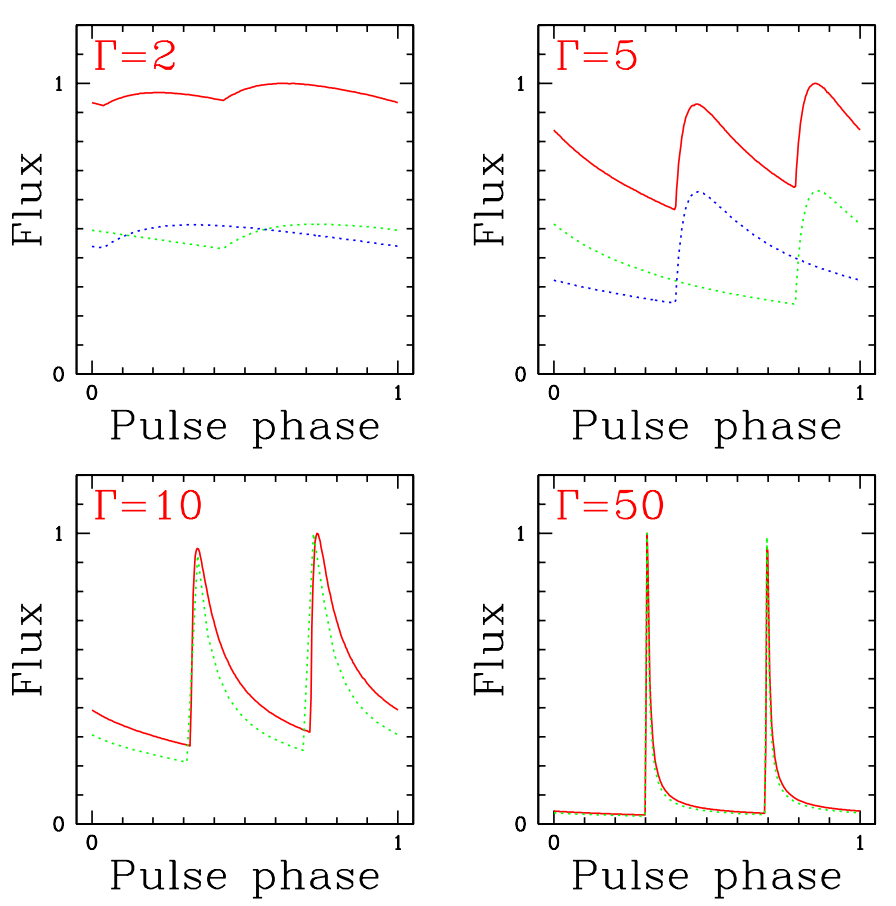

Fig. 2. Pulse shapes obtained by a numerical integration of Eq. (3). Four values of the Lorentz factor $(\Gamma)$ are shown, the remaining parameters are: $\alpha=60^{\circ}, \zeta=60^{\circ}, r_{0}=30 r_{\mathrm{L}}, a=0$ and $q=3$ (see text). The total emission is shown as a solid line. For $\Gamma=2$ and 5 , the contributions of the current sheets of different polarity are shown as dotted lines. For $\Gamma=10$ and 50, the dotted line shows the effect of a more rapid fading of the emissivity to larger radius $(q=6)$.

additional radio components (Moffatt \& Hankins 1996), one of which displays "core emission" properties (Rankin 1990). This suggests emission from close to the polar cap, with the magnetic axis passing within a few degrees of the line of sight. The core emission is in the precursor of the main pulse, which constrains the relative phase of the polar cap emission and wind emission in this interpretation. However, a complete solution linking the inner magnetosphere and the wind structure would be required to check this point. Nevertheless, core emission indicates that the inclination angle $\alpha$ of the magnetic axis to the rotation axis roughly equals the angle $\zeta$ between the rotation axis and the line of sight. The latter is determined by X-ray and optical observations of the "torus" (Aschenbach \& Brinkmann 1975; Hester et al. 1995) to be $60^{\circ}$. In Fig. 2 the emission has been assumed to switch on at $r_{0}=30 r_{\mathrm{L}}$ and pulses appear for $\Gamma>5$, in rough agreement with the estimate (1). At higher Lorentz factors, the pulses sharpen. From Eq. (2) it can be seen that the emission splits naturally into two contributions, corresponding to different signs of the term $\arccos (-\cot \alpha \cot \theta)$. In the first two plots of Fig. 2 these contributions are plotted separately as dotted lines. Although the detailed pulse shapes will depend on the structure of the reconnecting sheets in the Crab wind, as well as the details of the switching on mechanism and the amount of acceleration of the wind, the separation of the two peaks is not sensitive to these unknowns, being determined by the location of the sheets. This separation in phase equals $\arccos (\cot \alpha \cot \zeta) / 180^{\circ}$, corresponding to 0.39 for the Crab pulsar, in good agreement with observation (e.g., Kanbach 1998; Kuiper et al. 2001).

For the Vela pulsar, conflicting interpretations of the polarisation sweep and the $\mathrm{X}$-ray morphology give $\alpha \approx 71^{\circ}, \zeta \approx 65^{\circ}$ (Radhakrishnan \& Deshpande 2001) or $\alpha \approx \zeta \approx 53^{\circ}$ (Helfand et al. 2001). These imply separations of the gamma-ray (sub-)pulses of 0.45 and 0.31 of a period respectively, so that the observed spacing of 0.4 marginally favours the former interpretation.

\section{Luminosity estimates}

Electrons heated in the current sheets will enter the surrounding magnetic field and emit synchrotron radiation. An estimate of the power radiated can be found by assuming a monoenergetic electron population of Lorentz factor $\gamma(\gg 1)$ and requiring pressure equilibrium between the hot plasma and the surrounding magnetic field. In the delta-function approximation for the synchrotron emissivity, the luminosity at frequency $\nu$ into a solid angle $\Omega$ is

$\mathrm{d} L / \mathrm{d} \nu \mathrm{d} \Omega \approx \int_{r_{0}}^{\infty} \mathrm{d} r r^{2} \Delta \Gamma n_{\mathrm{h}}^{\prime} P_{\text {s.p. }} \delta\left(\nu-\nu_{0}\right)$

(Skjæraasen \& Kirk 2001), where $\Delta(\leq 1)$ is the fraction of a wavelength of the wind pattern occupied by hot electrons of proper density $n_{\mathrm{h}}^{\prime}, P_{\text {s.p. }}=(4 / 3) c \sigma_{\mathrm{T}} \gamma^{2}\left(B^{\prime 2} / 8 \pi\right)$ is the synchrotron power emitted by a single electron, and $\nu_{0}$ is the characteristic frequency of emission, measured in the observer frame ( $\sigma_{\mathrm{T}}$ is the Thomson cross section). Pressure equilibrium implies $n_{\mathrm{h}}^{\prime} \gamma m c^{2} / 3=B^{\prime 2} / 8 \pi$, where $B^{\prime}$ is the magnetic field in the comoving frame and $m$ the electron mass. Well outside the light cylinder, but before energy release begins, i.e., at $r_{\mathrm{L}}<r<r_{0}$, the density and magnetic field scale as $r^{-2}$ and $r^{-1}$ respectively, and can be characterised by the values $n_{\mathrm{L}}$ and $B_{\mathrm{L}}$ obtained by extrapolating back to $r=r_{\mathrm{L}}$. Provided the flow does not accelerate significantly whilst emitting synchrotron radiation, these same scalings, which imply $\gamma$ independent of $r$, can be used in the integration in Eq. (4). This is convenient for an estimate of the luminosity, but is sensitive to the assumption that the wind does not accelerate upon reconnection.

If we identify the ratio of Poynting flux to kinetic energy (at the light cylinder) $\sigma_{\mathrm{L}}=B_{\mathrm{L}}^{2} /\left(4 \pi \Gamma n_{\mathrm{L}} m c^{2}\right)$, define an average fraction of hot particles $\bar{\Delta}=$ $r_{0} \int_{r_{0}}^{\infty} \mathrm{d} r \Delta / r^{2} \lesssim 1$, and use the standard relation $B_{\mathrm{L}}=$ $2.9 \times 10^{8} \dot{P}^{1 / 2} P^{-5 / 2} \mathrm{G}$, the luminosity becomes

$$
\frac{\mathrm{d} \hat{L}}{\mathrm{~d} \Omega}=3.9 \times 10^{7}\left(\frac{\sigma \bar{\Delta} r_{\mathrm{L}} \dot{P}}{\Gamma^{3} r_{0} P^{4}}\right)(P \text { in } \mathrm{s})
$$

where $\mathrm{d} \hat{L} / \mathrm{d} \Omega$ is the synchrotron luminosity per steradian divided by the wind luminosity per steradian. Similarly, the characteristic frequency is

$h \nu_{0}(r)=2.6 \times 10^{-6}\left(r_{\mathrm{L}} / r\right) \sigma^{2} \dot{P}^{1 / 2} P^{-5 / 2} \mathrm{MeV}$. 
The spectrum of this model with monoenergetic electrons extends to a maximum frequency given by Eq. (6) with $r=r_{0}$ and is flat: $\mathrm{d} L / \mathrm{d} \nu \mathrm{d} \Omega \propto \nu^{0}$. However, this aspect is sensitive to expansion and acceleration of the plasma after reconnection. If, in a more realistic scenario, the electron density is a power law $N(\gamma) \propto \gamma^{-p}$, with $p>2$, then most of the energy resides in the particles with lowest $\gamma$ and the above estimate (6) refers to the photons emitted by these particles. The emission at frequencies $\nu<\nu_{0}\left(r_{0}\right)$ is dominated by the same low energy particles radiating at larger radius in a weaker magnetic field, and is determined by the expansion and acceleration of the hot plasma. In the absence of acceleration, the flat spectrum derived above prevails. However, the intrinsic spectrum, which is a power law $\mathrm{d} L / \mathrm{d} \nu \mathrm{d} \Omega \propto \nu^{-a}$, with $a>0.5$, is revealed at frequencies $\nu>\nu_{0}\left(r_{0}\right)$, where particles radiating at $r_{0}$ dominate. In the case of the Crab pulsar, the optical to $\mathrm{X}$-ray emission is consistent with a flat spectrum (Shearer \& Golden 2001), which steepens to $a>1$ at a photon energy of approximately $1 \mathrm{MeV}$ (Kanbach 1998; Kuiper et al. 2001). Equation (6) then implies $\sigma=1.1 \times 10^{4} \sqrt{r_{0} / r_{\mathrm{L}}}$. Observations suggest $\mathrm{d} \hat{L} / \mathrm{d} \Omega \approx 10^{-3}$, which, from Eq. (5), is consistent with a mildly supersonic flow, $\Gamma \approx 250$, that starts to radiate at $r_{0}=30 r_{\mathrm{L}}$. This satisfies the lower limit of $5 r_{\mathrm{L}}$ (Bogovalov \& Aharonian 2000) on the radius at which Poynting flux can be converted to particle-borne energy flux.

\section{Discussion}

Current models of the high energy emission from rotation powered pulsars fall into two groups: "polar cap" and "outer gap" models (see Harding 2001). Each locates the emission region in the corotating magnetosphere of the star. The predicted pulse shapes depend sensitively on the uncertain geometry of the magnetic field in this region, usually assumed dipolar. In contrast, in our model, pulses are emitted outside the corotation region. If the pulsar drives a supersonic, MHD wind, the field geometry there approaches a simple asymptotic solution (Bogovalov 1999), determining the basic properties of the pulses.

Pacini (1971) and Pacini \& Salvati (1983; Pacini \& Salvati 1987) suggested that particles close to the light cylinder emit synchrotron radiation and are responsible for the optical pulses of the Crab and other pulsars, a theory which appears to be in reasonable agreement with observation (Shearer \& Golden 2001). Our model is similar in that the radiation mechanism is synchrotron emission and the scaling of Eq. (5) is close to that originally given by Pacini (1971). It goes further, however, by specifying for the emission region a precise geometrical structure and location.

It is known that the dilution of the plasma in the wind must lead to non-ideal MHD behaviour (Usov 1975; Michel 1982) and so can be responsible for triggering the emission. The position at which this occurs depends on the initial concentration of charges in the current sheet and is taken as a free parameter. The major uncertainty in the model is the speed with which dissipation proceeds. Our computations implicitly assume rapid dissipation (over a scale small compared to the radius). In this case, it is possible to make a rough estimate of the synchrotron luminosity which is in agreement with observations. It is conceivable that at least part of the radio emission could be produced in the wind region, as is suggested by the similarity of the pulse profiles at all frequencies seen in the Crab pulsar. However, until a candidate coherent mechanism can be identified, this connection remains very speculative. Nevertheless, the wind scenario is a viable alternative to current theories of gamma-ray, X-ray and optical pulses from rotation-driven pulsars and is capable of making testable predictions about the pulse profile.

Acknowledgements. This work is a collaboration of the TMR Network "Astroplasmaphysics" of the European Commission, contract FMRX-CT-98-0168. Y.G. is supported by a Marie Curie Fellowship from the European Community, contract no. HPMFCT-2000-00671 under the IHP programme.

\section{References}

Arons, J. 1996, Space Sci. Rev., 75, 235

Aschenbach, B., \& Brinkmann, W. 1975, A\&A, 41, 147

Bogovalov, S. V. 1999, A\&A, 349, 1017

Bogovalov, S. V., \& Aharonian, F. A. 2000, MNRAS, 313, 504

Coroniti, F. V. 1990, ApJ, 349, 538

Crusius-Wätzel, A. R., Kunzl, T., \& Lesch, H. 2001, ApJ, 546, 401

Harding, A. K. 2001, in High energy gamma-ray astronomy, ed. F. Aharonian, \& H. J. Völk, AIP

Helfand, D. J., Gotthelf, E. V., \& Halpern, J. P. 2001, ApJ, 556,380

Hester, J. J., Scowen, P. A., Sankrit, R., et al. 1995, ApJ, 448, 240

Kanbach, G. 1998, Adv. Space Res., 21, 227

Kirk, J. G., \& Lyubarsky, Y. 2001, Publ. Astron. Soc. Aust., 18,415

Kuiper, L., Hermsen, W., Cusumano, G., et al. 2001, A\&A, 378, 918

Lyubarsky, Y. 1996, A\&A, 311, 172

Lyubarsky, Y., \& Kirk, J. G. 2001, ApJ, 547, 437

Machabeli, G. Z., Luo, Q., Melrose, D. B., \& Vladimirov, S. 2000, MNRAS, 312, 51

Melatos, A. 1998, Mem. Soc. Ast. It., 69, 1009

Michel, F. C. 1982, Rev. Mod. Phys., 54, 1

Michel, F. C. 1994, ApJ, 431, 397

Michel, F. C., \& Li, H. 1999, Phys. Rep., 318, 227

Moffatt, D. A., \& Hankins, T. H. 1996, ApJ, 468, 779

Pacini, F. 1971, ApJ, 163, L17

Pacini, F., \& Salvati, M. 1983, ApJ, 274, 369

Pacini, F., \& Salvati, M. 1987, ApJ, 321, 447

Radhakrishnan, V., \& Deshpande, A. A. 2001, A\&A, 379, 551

Rankin, J. M. 1990, ApJ, 352, 247

Skjæraasen, O., \& Kirk, J. G. 2001, in Similarities and universality in relativistic flows, ed. Georganopoulos, Guthmann, Manolakou, \& Marcowith (Logos Verlag, Berlin)

Shearer, A., \& Golden, A. 2001, ApJ, 547, 967

Usov, V. V. 1975, Astrophys. \& Space Sci., 32, 375

Zenitani, S., \& Hoshino, M. 2001, ApJ, 562, L63 